\section{El aprendizaje autorregulado en el PLE a través de una estrategia didáctica basada en portafolios electrónicos con blogs y microblogs}

UTE. Revista de Ciències de I'Educació

Monogràfic 2020. Pag. 83-101

ISSN 1135-1438. EISSN 2385-4731

http://revistes.publicacionsurv.cat/index.php/ute

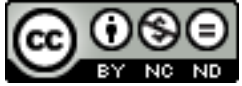

https://doi.org/10.17345/ute.2020.4.2799

\author{
Gemma Tur $\mathbb{D}$, Urith Ramírez-Mera \\ Rebut: 25/04/2020 Acceptat: 04/11/2020
}

\begin{abstract}
Resumen
Los Entornos Personales de Aprendizaje (PLE) han sido relacionados con el aprendizaje autorregulado al resaltarse sus posibilidades para la autonomía en el propio proceso de aprendizaje así como por la incidencia en las habilidades metacognitivas de planificación, ejecución y reflexión. De entre todas las plataformas, los blogs y microblogs han sido protagonistas por su flexibilidad y capacidad de adaptación a numerosos diseños, entre ellos los portafolios electrónicos. La investigación ha mostrado las posibilidades de Blogger para la creación de portafolios reflexivos y Twitter para la ampliación de redes personales de aprendizaje. En esta experiencia se propone una actividad compleja y global en la que ambas plataformas se abordan para la construcción de un portafolio electrónico que contempla una fase armónica de cohesión reflexiva y otra más más distribuida de reflexión compartida y en red. Con un enfoque interpretativo, se analizan los diagramas del PLE del alumnado y se recogen sus percepciones a través un cuestionario en línea. Los resultados muestran que la estrategia es válida y que permite el desarrollo de habilidades metacognitivas de planificación y ejecución con ambas plataformas aunque las habilidades reflexivas se desarrollan preferentemente en blogs.
\end{abstract}

Palabras claves: Entorno Personal de Aprendizaje, blog, microblog, portafolio electrónico, aprendizaje autorregulado

\begin{abstract}
Personal Learning Environments (PLE) have been related to self-regulated learning by highlighting their possibilities for autonomy in one's own processes as well as by influencing metacognitive skills of planning, execution and reflection. Among all the platforms, blogs and microblogs have been the protagonists due to their flexibility and capacity to adapt to numerous designs, and electronic portfolios among them. Research has shown the possibilities of Blogger for the creation of reflective portfolios and Twitter for the extension of personal learning networks. In this experience, a complex and global activity is proposed in which both platforms are approached for the construction of an electronic portfolio that contemplates a harmonic phase of reflexive cohesion and another more distributed phase of shared and networked reflection. With an interpretative approach, the PLE diagrams by students are analyzed and their perceptions are collected through an online questionnaire. The results show that the strategy is valid and that it allows the development of metacognitive planning and execution skills with both platforms, although the reflective ones are developed preferably in blogs.
\end{abstract}

Key words: Personal Learning Environment, blog, microblog, electronic portfolio, self-regulated learning. 


\section{Introducción}

La introducción de las Tecnologías de la Información y la Comunicación (TIC) en el contexto educativo ha generado la creación de ecosistemas de aprendizaje cuya complejidad y ubicuidad difieren, por mucho, de la educación tradicional que se lleva a cabo dentro del aula; el desarrollo de habilidades y destrezas evoluciona, y en consecuencia, la forma de enseñar y aprender (Pozos y Tejeda, 2018). Entre algunos modelos didácticos que permiten al alumnado tener una mayor autonomía y control en su proceso de enseñanza-aprendizaje está el enfoque basado en entornos personales de aprendizaje, el cual ha sido recurrente en la formación docente inicial (García-Martínez et al., 2020; Korhonen et al., 2018), con la finalidad añadida de la proyección en su futura docencia (Kramarski y Michalsky, 2010), ya que permite el desarrollo de habilidades y conocimientos que serán transmitidas a sus educandos.

En una reciente revisión sistematizada de literatura sobre educación se observa que el concepto PLE está estrechamente ligado al del aprendizaje autorregulado (Castañeda et al., 2019). El estudio observa que el concepto de aprendizaje autorregulado en los PLE en muchos casos se asocia con la social media, que permiten la propiedad y control por parte de la persona que aprende. Por otra parte, cabe destacar que en los diseños de fomento de los PLE en entornos formales, los portafolios emergen como la estrategia didáctica más genuina (Castañeda et al., 2019), que ha sido especialmente bien aceptada en la formación docente inicial (Soria y Carrió, 2016).

\section{Marco teórico}

\subsection{Los Entornos Personales de Aprendizaje y el aprendizaje autorregulado}

Los Entornos Personales de Aprendizaje o PLE (Personal Learning Environments) son espacios centrados en el alumno (Castañeda y Adell, 2013) que se relacionan con el autoaprendizaje (Chaves-Barboza et al., 2019; Romero et al., 2019; Araka et al., 2020) y el aprendizaje a lo largo de la vida (Blaschke, 2019) que busca que el alumno tome control de su proceso de aprendizaje y que, de manera más específica, apoye al desarrollo de la agencia del alumno. En trabajos muy recientes se reconoce el valor de los PLE para el aprendizaje personal y personalizado (Fiedler y Väljataga, 2020) y su característica eminentemente sociomaterial (Dabbagh y Castañeda, 2020).

Los PLE, más allá de la perspectiva tecnodeterminista, son ecosistemas abiertos (Kühn, 2017; Panagiotidis, 2012) que conectan herramientas, servicios, estrategias, relaciones e interacciones al proceso de aprendizaje (Barroso et al., 2012; Marín et al., 2013) dentro de contextos escolarizados y no escolarizados (Dabbagh y Kitsantas, 2012; Area y Sanabria, 2014). Los PLE siguen un sustento pedagógico que integra el aprendizaje autorregulado o SRL (Self-Regulated Learning) (Perera y Gardner, 2018; Castañeda et al., 2019) y el aprendizaje social (Dabbagh y Kitsantas, 2012; Nkwenti, 2016) y que siguen un paradigma socioconstructivista, constructivista y conectivista (Elia y Poce, 2010; Torres et al., 2019).

Los PLE se han relacionado con el aprendizaje autorregulado y de entre todos los modelos, el modelo de Zimmerman (2002) ha sido recurrente (Dabbagh y Kitsantas, 2012; Yen et al., 2016). Este modelo describe el SRL en tres fases, cada una de ellas con dos subfases. La primera fase es la planificación que incluye las estrategias metacognitivas relacionadas con la organización previa de la tarea, planeación y la actitud de automotivación. En la segunda fase de realización, se implementa el plan elaborado previamente mientras se monitoriza y optimiza según los diferentes estilos de aprendizaje. La autorreflexión, que es la tercera fase, permite que el estudiante reflexione sobre su aprendizaje, el nivel 
conseguido respecto de la norma y, el aprendizaje de otros, aspecto que permite el desarrollo de actitudes que pueden promover o limitar aprendizajes futuros. Para Zimmerman (2002) las habilidades metacognitivas del modelo de aprendizaje autorregulado pueden ser aprendidas, y de hecho se suelen desarrollar en contextos sociales y formales desde edades tempranas.

\subsection{Los portafolios electrónicos con blogs y microblogs en el contexto del PLE}

El uso de portafolios, tanto si son en papel como si son electrónicos, suele basarse en tres principales procesos: la documentación del aprendizaje, la reflexión y el trabajo colaborativo entre pares y profesorado (Zubizarreta, 2009). Sin embargo, en entornos digitales, la colaboración con otros se hace más evidente, ampliando sus posibilidades (Cebrián, 2011) más allá del grupo de pares en la educación formal y posibilitando procesos colaborativos de diferente naturaleza, tanto para el desarrollo de habilidades digitales como de otras de tipo metacognitivo (Tur y Urbina, 2016).

La aportación de Cambridge (2010) a la conceptualización de los portafolios electrónicos (o eportafolios) es relevante en el contexto de los entornos sociales (Cobos-Sanchiz et al., 2016) puesto que describe dos procesos reflexivos de diferentes características según su conexión en red. Así, Cambridge (2010) define los procesos del yo sinfónico (symphonic self) como aquellos de mayor metacognición, individuales y de interrelación con las evidencias del aprendizaje realizado. A esta identidad más introspectiva del eportafolio, añade otra que denomina como el yo en red (networked self), también reflexiva, pero más rápida, menos profunda y más conectada y en red, que permite ampliar horizontes y hacer relaciones que amplían el aprendizaje. Este segundo proceso es al que Cambridge (2010) atribuye a la influencia de la social media, mientras que el yo sinfónico sería el que corresponde al estilo de portafolio más auténtico.

La realidad de la práctica educativa muestra cómo los blogs, plataformas de social media por antonomasia desde los inicios de la web 2.0, se han convertido en los servicios más recurrentes para la creación de portafolios electrónicos (Marín, 2020), por lo que siguen permitiendo procesos más reflexivos, profundos e introspectivos. Consecuentemente, la actividad reflexiva del yo en red, breve y rápida, se puede desarrollar en otros servicios que permitan este formato. Tal es el caso, de los servicios de microblogs, de entre los cuales, Twitter ya ha sido analizado desde sus posibilidades para la formación docente, y la promoción de la reflexión gracias a su formato corto (Carpenter et al., 2016; Tur et al., 2017).

Tanto los blogs como los microblogs se relacionan con el SRL. Así, en relación a los blogs, la investigación ha observado sus posibilidades para la reflexión (Biberman-Shalev, 2018), la co-evaluación (Dippold, 2009), y en general, las habilidades metacognitivas de autorregulación del aprendizaje (Hatzipanagos y Warburton, 2009; Martínez et al., 2015). Sobre Twitter, en su modelo de interrelación de PLE y SRL, Dabbagh y Kitsantas (2013) observan que se usa más para la gestión de la información y la colaboración que para la autoevaluación, lo cual es discutido en investigaciones posteriores. Por ejemplo, Cho y Cho (2013) destacan el valor de Twitter para la planificación y la reflexión, y Pérez-Garcías et al. (2018) observan que el uso de Twitter permite la monitorización durante la ejecución del aprendizaje así como la posterior reflexión.

Sin embargo, a pesar de estos estudios, aún se requiere una estrategia más detallada, que permita conocer con mayor profundidad cómo el SRL se optimiza en entornos virtuales y cómo éstos promueven las habilidades metacognitivas según sus posibilidades. Este artículo presenta una propuesta didáctica, compleja y global, en la que se propone el desarrollo del PLE del alumnado, centrado en una estrategia con portafolios electrónicos con el uso de plataformas sociales para promover el análisis metacognitivo según el modelo del SRL. 


\section{Investigación}

\subsection{Contexto}

Esta investigación se realiza a partir de una actividad de aprendizaje realizada en el Máster de Formación del Profesorado de la Universidad de las Islas Baleares, en la Sede Universitaria de Ibiza y Formentera. La actividad está conformada por tres partes: la primera parte implica el fomento de las TIC para el acceso, la creación y la compartición de conocimiento (Castañeda y Adell, 2013), cuyo análisis metacognitivo se centra en una estrategia didáctica con portafolios que incluye dos actividades de diferente naturaleza con blogs y microblogs (segunda parte), y una tercera parte de reflexión y explicitación del entorno personal de aprendizaje generado por el alumno (Figura 1). Por tanto, la actividad tiene un triple objetivo: a) primero, fomentar la generación de entornos de aprendizaje enriquecidos con TIC, b) incidir en la documentación del aprendizaje con portafolios electrónicos, y c) ayudar a hacer consciente al alumnado de su propio PLE y de las estrategias de autorregulación del aprendizaje que llevan a cabo.

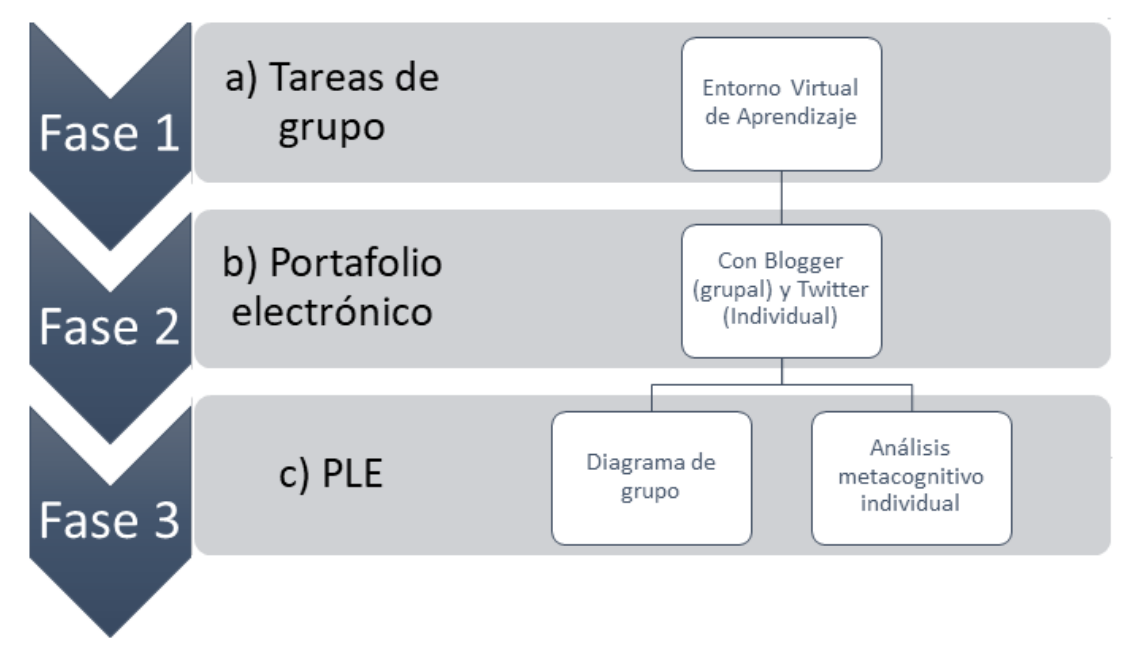

Figura 1. Fases del diseño de la actividad

Los participantes realizan varias tareas cíclicas en cada una de las fases. En la Fase 1 (con tareas de grupo), se realiza una documentación del aprendizaje que se desarrolla en actividades colaborativas de diferente estructura grupal (jigsaw con diferentes agrupaciones por cada iteración y grupos estables a lo largo del curso), con una presentación oral en clase presencial. El alumnado, en la Fase 2 (eportafolio), desarrolla dos procesos reflexivos diferentes basados en Cambridge (2010): el primero, grupal y de escritura reflexiva en blogs; $y$, un segundo, con el uso de Twitter para el análisis crítico desde una perspectiva individual de la metacognición grupal, y la lectura y contraste con el aprendizaje de los pares. Así, la creación de portafolios en blogs obedece a la conceptualización del yo sinfónico mientras que el yo en red se materializa con la actividad reflexiva más dinámica, breve y directamente conectada con otros en Twitter. La secuencia didáctica de las fases 1 y 2 se detalla en la Figura 2. 


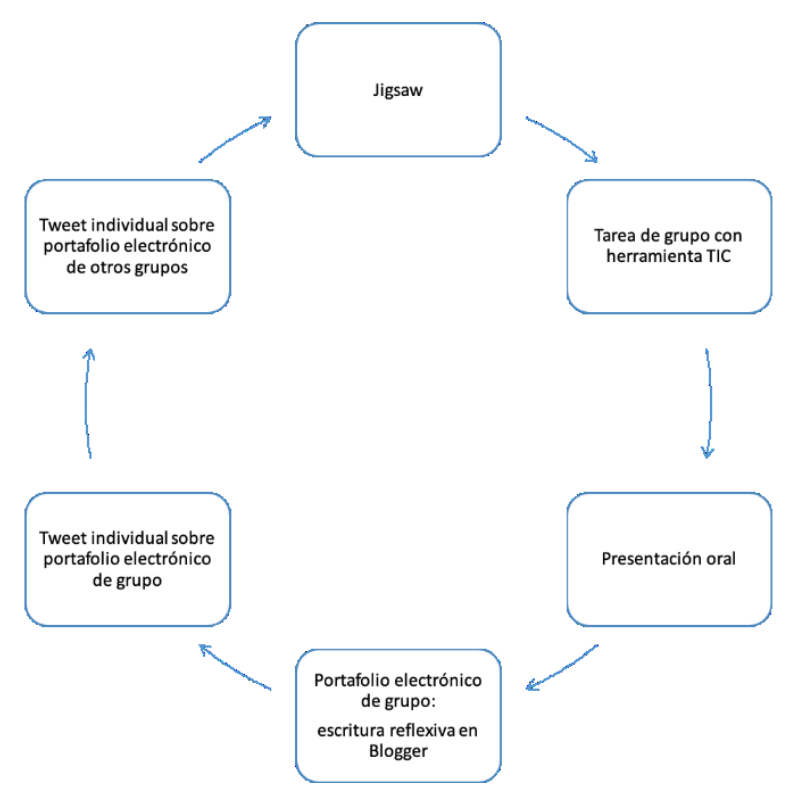

Figura 2. Diagrama de la secuencia didáctica de la Fase 1 y Fase 2.

En la fase final de la estrategia didáctica, el alumnado hace una representación de su PLE grupal en diagramas siguiendo la conceptualización de Castañeda y Adell (2013) sobre los componentes del PLE, y que además incluye su relación con el aprendizaje autorregulado (Zimmerman, 2002) (Figura 3), es decir, estos dos elementos se integran de manera paralela al proceso de aprendizaje. El diagrama de los PLE grupales se acompaña de textos reflexivos que profundizan en los diversos procesos del SRL haciendo especial énfasis en las fases de autorregulación con blogs y microblogs, así como en su incidencia en su futura labor docente.

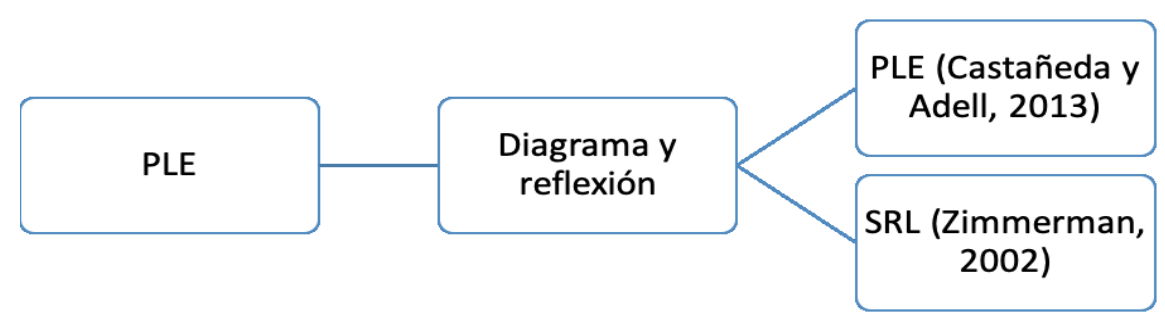

Figura 3. Construcción de PLE

\subsection{Participantes}

El alumnado que realizó la actividad que se presenta comprende los grupos de las promociones de tres cursos desde 2016-17 hasta el curso 2018-19 con un total de 61 alumnos, de los cuales 47 alumnos participaron través de un cuestionario de investigación - 19 de género masculino y 28 de género femenino, de entre 18 y 29 años principalmente.

\subsection{Objetivos de investigación}

Esta investigación tiene el siguiente objetivo general: 
- Explorar la autorregulación del alumnado en sus Entornos Personales de Aprendizajes o PLE a través de una estrategia didáctica de portafolio electrónico con blogs y microblogs para su formación docente inicial.

Para ello se plantean los siguientes dos objetivos específicos:

- Analizar las percepciones del alumnado sobre la aplicación de los dos procesos de portafolio con blogs y microblogs según las fases del modelo de aprendizaje autorregulado

- Explorar el uso de los dos procesos de portafolio con blogs y microblogs en los diagramas según su rol en la interrelación entre el PLE y el enfoque para el aprendizaje autorregulado

\subsection{Metodología}

En esta investigación se llevó a cabo una metodología mixta, pues debido a las características de la investigación, es necesario utilizar diseños multimodales (Creswell y Clark, 2007). Además, permite la recolección de datos empíricos a través de datos numéricos y cualitativos con el objetivo de reunir datos redondeados y confiables (Cohen et al., 2007). El uso de cuestionarios permite obtener una imagen general, dado que su finalidad es descriptiva y corresponde a un primer acercamiento al tema investigado (Torrado, 2009), y se logra un análisis más detallado y profundo a través de información cualitativa recogida a través de datos cualitativos (Sabariego et al., 2009).

Se llevó a cabo un diseño de investigación con triangulación concurrente o transformativo secuencial (DITRAS) (Cedeño, 2012). Este modelo incluye dos etapas de recolección de datos. La prioridad y fase inicial puede ser cuantitativa o cualitativa, y los resultados de ambas etapas son integrados durante la interpretación. Además, permite tener una teoría, marco conceptual o ideología clara que determina la dirección de la investigación (Creswell y Clark, 2007).

Para la investigación cuantitativa se implementó un cuestionario pues es un instrumento que permite recopilar información estructurada (Wilson y McLean, 1994), y busca conocer la percepción del alumno con respecto a las herramientas que se utilizan para la creación de eportafolio y su integración en el proceso de autorregulación. Posteriormente, para la investigación cualitativa se realizó un análisis de contenidos, ya que representa un estricto y sistemático conjunto de procedimientos para el análisis, examen y verificación de los contenidos de datos escritos (Flick, 1998; Mayring, 2004), pues los textos y los diagramas que el alumno realiza pueden ser leídos, interpretados y entendidos (Krippendorp, 2004). Finalmente, se analizaron los datos tomando en consideración los resultados del cuestionario y el desarrollo del PLE a través de diagramas y las reflexiones del profesorado en formación.

\subsection{Instrumento}

Para la primera parte de la investigación se utilizó un enfoque cuantitativo de corte transversal. Para la recolección de los datos se desarrolló un cuestionario ad hoc con seis secciones con base en el modelo de SRL de Zimmerman (2002): planificar (análisis de tareas y creencias auto-motivacionales), ejecutar (auto-observación y auto-control), y autorreflexión (auto-juicio y auto-reacción). En cada una de las secciones, se hace referencia a la incidencia que tienen las herramientas digitales Blogger y Twitter en cada fase del SRL. Para comprobar la confiabilidad del instrumento, se realizaron pruebas de fiabilidad, y se obtuvo un alfa de Cronbach de .926, que representa una alta confiabilidad del instrumento (Cervantes, 2005). En la Tabla 1 se presentan las preguntas que componen el instrumento utilizado. 
El aprendizaje autorregulado en el PLE a través de una estrategia didáctica basada en portafolios con blogs y microblogs

Tabla 1: Instrumento de investigación

\begin{tabular}{|c|c|c|}
\hline Fases del SRL & Proceso & Pregunta \\
\hline \multirow[t]{2}{*}{ Planificación } & Análisis de tareas & $\begin{array}{l}\text { ¿La herramienta/actividad me ha ayudado a definir mis objetivos y hacer } \\
\text { una planificación estratégica? }\end{array}$ \\
\hline & $\begin{array}{l}\text { Creencias auto } \\
\text { motivadoras }\end{array}$ & $\begin{array}{l}\text { ¿La herramienta/actividad me ha ayudado a ser consciente de mis } \\
\text { capacidades desarrollando expectativas sobre mis propios resultados? }\end{array}$ \\
\hline \multirow[t]{2}{*}{ Ejecución } & Auto-observación & $\begin{array}{l}\text { ¿La herramienta/actividad me ha ayudado a hacer el seguimiento de mi } \\
\text { propio aprendizaje? }\end{array}$ \\
\hline & Auto-control & $\begin{array}{l}\text { ¿La herramienta/actividad me ha ayudado a ser capaz de controlar mi } \\
\text { proceso de aprendizaje? }\end{array}$ \\
\hline \multirow[t]{2}{*}{ Reflexión } & Auto-Juicio & ¿La herramienta/actividad me ha ayudado a autoevaluar mi aprendizaje? \\
\hline & Auto-reacción & $\begin{array}{l}\text { ¿La herramienta/actividad me ha ayudado a desarrollar la satisfacción por } \\
\text { el aprendizaje? }\end{array}$ \\
\hline
\end{tabular}

Para el procesamiento de los datos se colocó una escala Likert de cinco valores, por ejemplo, el valor 1 corresponde a "muy en desacuerdo" mientras que el valor 5 representa estar "muy de acuerdo". Posteriormente los datos se analizaron a través del programa estadístico SPSS Statistics 22.

\section{Resultados}

\subsection{Percepciones del alumnado sobre uso de blog y microblog con base al aprendizaje autorregulado (SRL)}

Al revisar los datos, haciendo un análisis general y descriptivo de los resultados, se encuentra que en cada fase del modelo de SRL existe una preferencia por el uso de Blogger sobre Twitter. Específicamente, en la fase de Ejecución (Fase 2 ) el uso de Blogger es mayor $(\bar{X}=3.988 \sigma=1.047$ ), mientras que el uso de Twitter $(\bar{X}=3.00 \sigma=1.261)$ queda lejos de ser aceptado de manera importante. Con respecto a la fase de Planificación y Ejecución que integran el SRL, se obtiene que el uso de Blogger supera a Twitter de manera significativa (Tabla 2).

Tabla 2: Uso de Blogger y Twitter en las tres fases del aprendizaje autorregulado

\begin{tabular}{|c|c|c|c|c|c|c|}
\hline & \multicolumn{2}{|c|}{ Planificación (Fase 1) } & \multicolumn{2}{|c|}{ Ejecución (Fase 2) } & \multicolumn{2}{|c|}{ Autorreflexión (Fase 3) } \\
\hline & Blogger & Twitter & Blogger & Twitter & Blogger & Twitter \\
\hline Media $(\bar{X})$ & 3.883 & 3.108 & 3.988 & 3.000 & 3.872 & 2.858 \\
\hline \multicolumn{7}{|c|}{ Desviación estándar } \\
\hline$(\sigma)$ & 1.048 & 1.357 & 1.047 & 1.261 & 1.110 & 1.191 \\
\hline
\end{tabular}


En la Figura 4 se presenta un comparativo entre la elección de Twitter y Blogger en donde se observa que el uso de Blogger tiene mayor aceptación al realizar actividades relacionadas con el auto-control $(\cdot=4.11, \sigma=1.092)$, mientras que Twitter tiene una mayor aceptación para realizar actividades metacognitivas relacionadas con las creencias auto-motivacionales $(\cdot=3.18, \sigma=1.467)$ en comparación con otras fases del SRL. También se observa que para el análisis de tareas, Blogger tiene sus valores más bajos $(\bar{X}=3.76, \sigma=1.119)$ mientras que Twitter tiene valores relativamente altos en comparación con otras fases del SRL ( $\bar{X}=3.11, \sigma=1.301)$. Por otro lado, Twitter presenta valores más bajos cuando el alumno realiza actividades metacognitivas asociadas a la autorreflexión $(\cdot=3.02, \sigma=1.282)$.

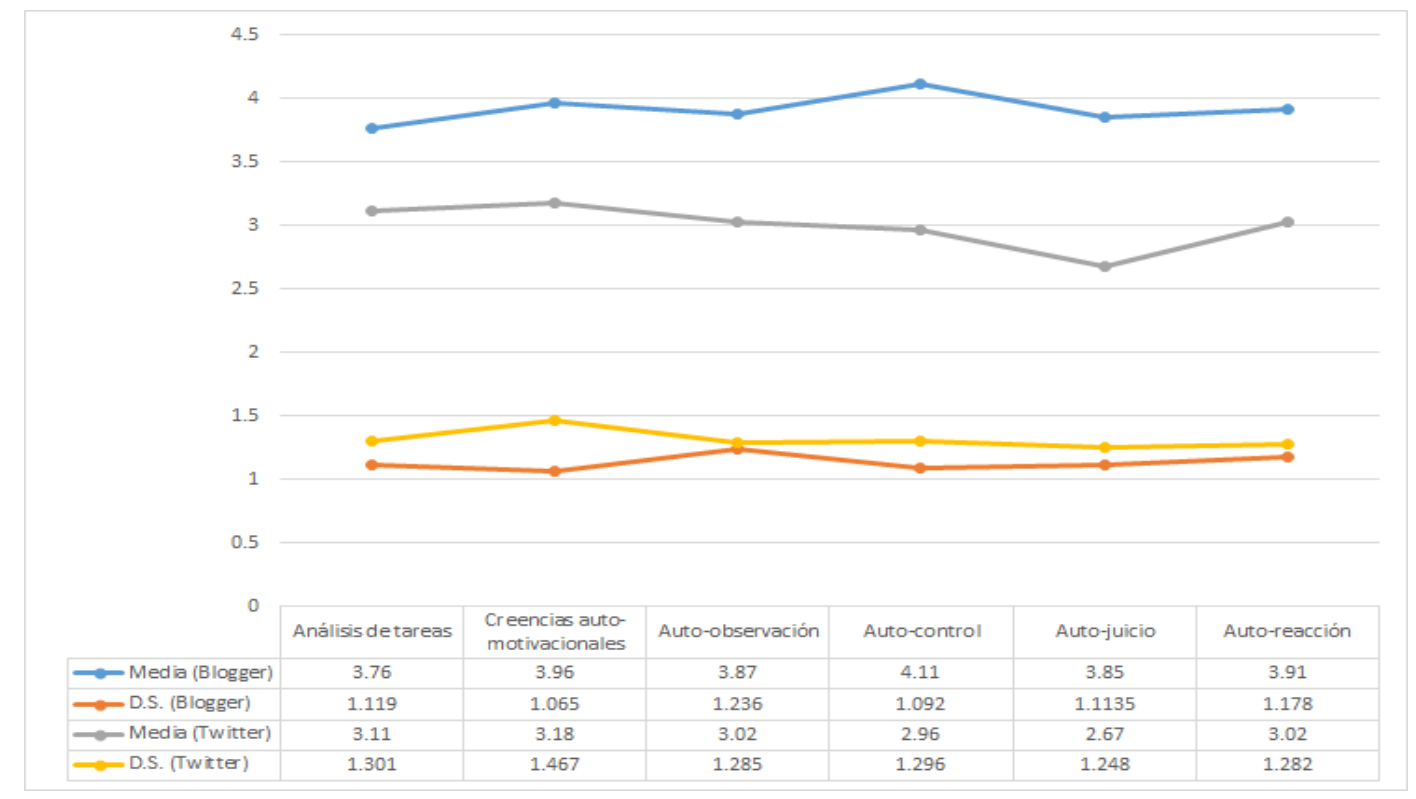

Figura 4. Uso de Blogger y Twitter en las subfases del SRL.

Dicho de otra manera, al menos el 73\% del alumnado está de acuerdo en que Blogger permite llevar a cabo su proceso de autoaprendizaje autorregulado. Sobre Twitter no más del $48 \%$ del alumnado está de acuerdo en que esta herramienta apoya su aprendizaje. Siendo observadores con este dato, en la Figura 5 se muestran las subfases en la Fase 2 (subfase de auto-control) y Fase 3 (subfase de auto-juicio) del SRL en la que Twitter presenta sus porcentajes más bajos.

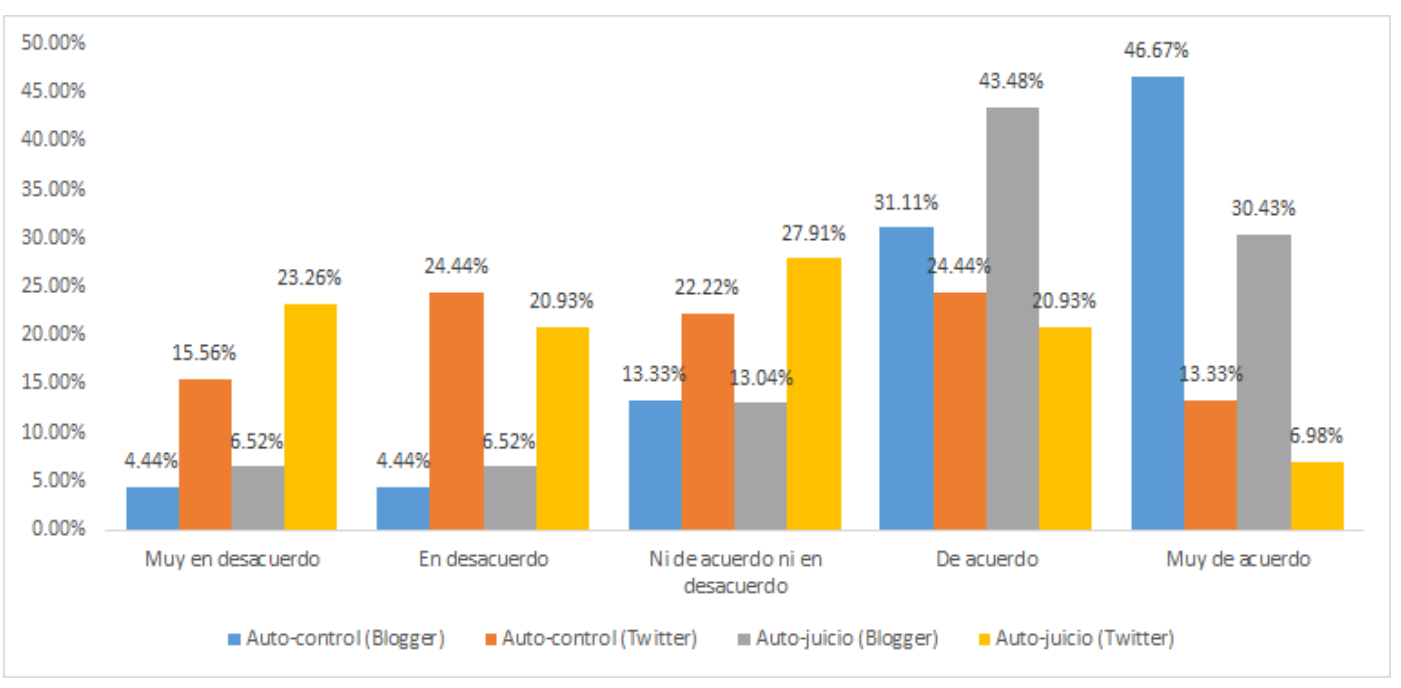

Figura 5. Comparación entre Blogger y Twitter en dos subfases del SRL 
Estos datos descriptivos no solo nos permiten comparar las dos plataformas del eportafolio del alumnado y su rol respecto del modelo de SRL sino que además nos ayudan a ver cómo el alumnado las percibe en cada fase. Por otro lado, siendo reservados con los datos que se exponen, se aplicaron pruebas de normalidad demostrando que la muestra es no paramétrica $(p<0.05)$. Por ello se realizó la prueba de correlación de Spearman.

Tabla 3: Correlaciones entre las fases del SRL y, Blogger y Twitter

\begin{tabular}{lllll}
\hline & & F1_Blogger & F2_Blogger & F3_Blogger \\
\hline F1_Blogger & Coeficiente de correlación & 1.000 & $.636^{\star \star}$ & $.800^{\star \star}$ \\
& Sig. (bilateral) & & .000 & .000 \\
& $\mathrm{~N}$ & 47 & 45 & 47 \\
\multirow{3}{*}{ F2_Blogger } & Coeficiente de correlación & $.636^{\star \star}$ & 1.000 & $.648^{\star \star}$ \\
& Sig. (bilateral) & .000 & & .000 \\
& $\mathrm{~N}$ & 45 & 45 & 45 \\
F3_Blogger & Coeficiente de correlación & $.800^{\star \star}$ & $.648^{\star *}$ & 1.000 \\
& Sig. (bilateral) & .000 & .000 & \\
& $\mathrm{~N}$ & 47 & 45 & 47 \\
\hline
\end{tabular}

\begin{tabular}{|c|c|c|c|c|}
\hline & & F1_Twitter & F2_Twitter & F3_Twitter \\
\hline \multirow[t]{3}{*}{ F1_Twitter } & Coeficiente de correlación & 1.000 & $.873^{\star \star}$ & $.911^{\star \star}$ \\
\hline & Sig. (bilateral) & & .000 & .000 \\
\hline & $\mathrm{N}$ & 46 & 45 & 46 \\
\hline \multirow{3}{*}{ F2_Twitter } & Coeficiente de correlación & $.873^{* *}$ & 1.000 & $.930^{\star \star}$ \\
\hline & Sig. (bilateral) & .000 & & .000 \\
\hline & $\mathrm{N}$ & 45 & 45 & 45 \\
\hline \multirow{3}{*}{ F3_Twitter } & Coeficiente de correlación & $.911^{\star \star}$ & $.930^{\star *}$ & 1.000 \\
\hline & Sig. (bilateral) & .000 & .000 & \\
\hline & $\mathrm{N}$ & 46 & 45 & 46 \\
\hline
\end{tabular}

En la Tabla 3 se observa que, de manera interna, existe correlación entre las fases que componen el SRL y Twitter, así como entre Blogger (sig.<0.01). 


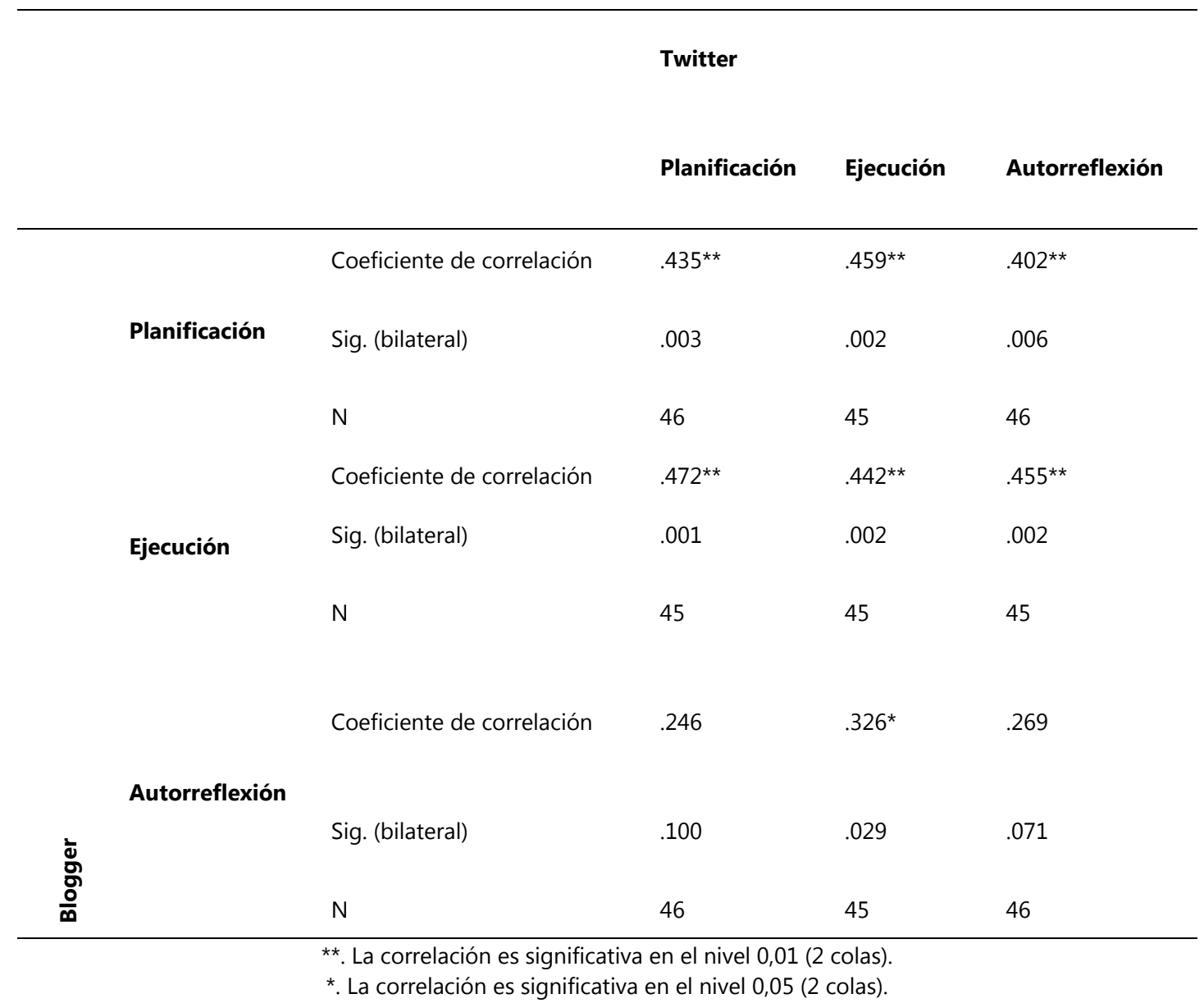

En la Tabla 4 se muestran la correlaciones que existen entre Twitter y Blogger en las distintas fases del aprendizaje autorregulado, resaltando que Twitter en la fase de planificación y reflexión no se asocia con Blogger en la fase de Reflexión del SRL.

\subsection{Blogger y Twitter en los diagramas de PLE del alumnado}

En la realización cíclica de tareas grupales, el alumnado va ampliando su PLE, según el modelo de Castañeda y Adell (2013), y al final de la programación didáctica, se plantea el análisis de ese PLE a través de la diagramación y la reflexión metacognitiva del conjunto. Esta actividad de diagrama, tiene un largo recorrido de implementación didáctica (por ejemplo, ver en Marin y Tur (2014), y se completa con la perspectiva añadida del modelo de Zimmerman (2002). Por lo tanto, la propuesta se amplía de manera que la reflexión metacognitiva sobre la planificación, ejecución y auto-reflexión del aprendizaje se refiere a los procesos de acceso, creación y colaboración propios del PLE.

En las tres promociones que se recogen en este estudio, las recomendaciones fueron mejorando gracias a la experiencia acumulada. Así en el primer año, se incentivaron representaciones gráficas creativas en las que las interrelaciones fueran potenciadas, pero a la vista de la dificultad del diagrama, en los dos siguientes cursos se permitió o bien una representación más creativa, o una más esquemática y secuenciada. Las figuras 6 y 7 muestran dos ejemplos de cada caso respectivamente, que corresponden a las promociones 17-18 y 18-19 y que coinciden en gran medida. En ambos casos, Twitter y Blogger fueron implementadas para acceder a la información implican la puesta en marcha de las tres actividades metacognitivas del modelo de SRL. En los procesos para crear el conocimiento, Twitter desaparece también de los dos diagramas diseñados por el alumnado, mientras que Blogger se mantiene con las 
UTE. Revista de Ciències de l'Educació Monogràfic 2020. Pàg. 83-101
El aprendizaje autorregulado en el PLE a través de una estrategia didáctica basada en portafolios con blogs y microblogs

tres fases de SRL. Para las actividades de compartir y colaborar, los dos diagramas coinciden en otorgar a Twitter las tres fases de SLR y, en cambio, Blogger no presentaría la fase de planificación.

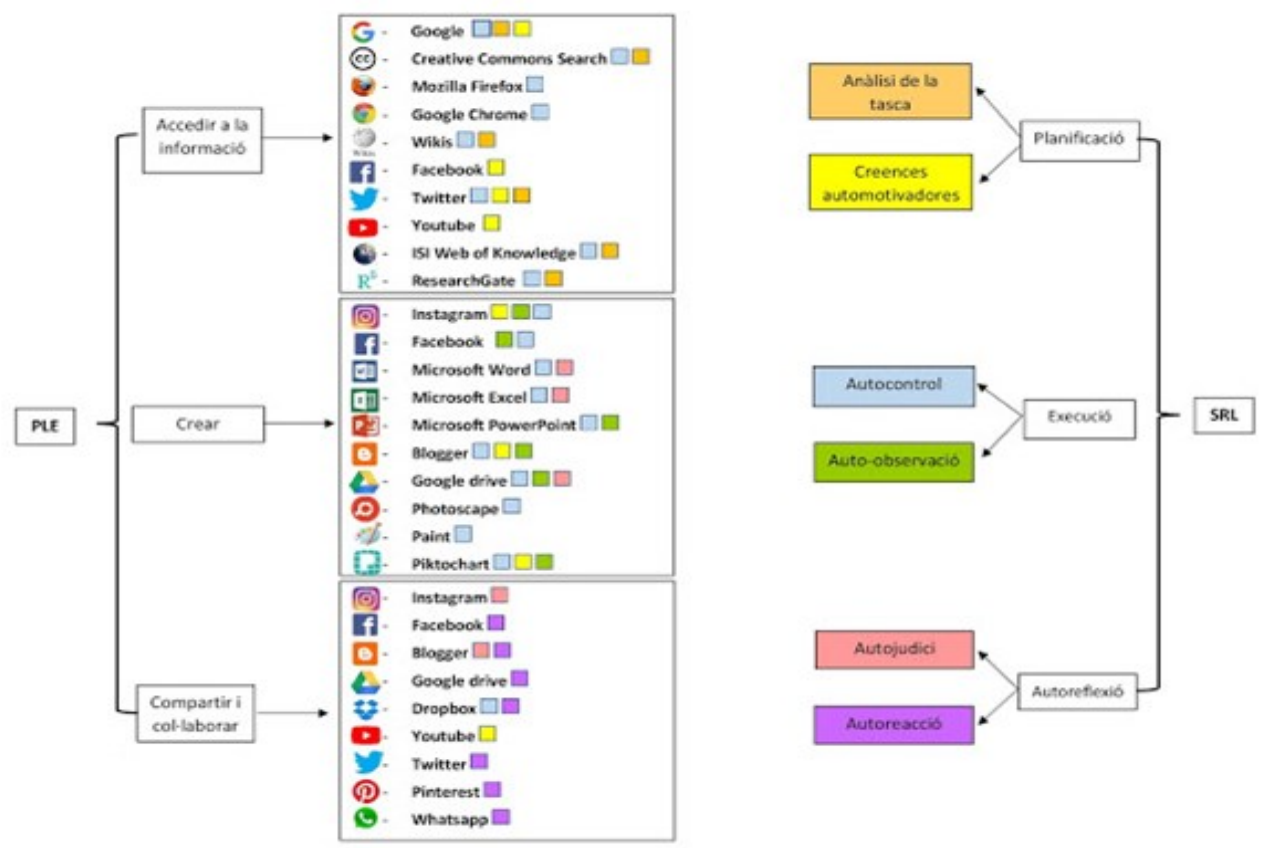

Figura 6. Ejemplo de diagrama de PLE con subfases del SRL. Grupo 1, Curso 17-18a
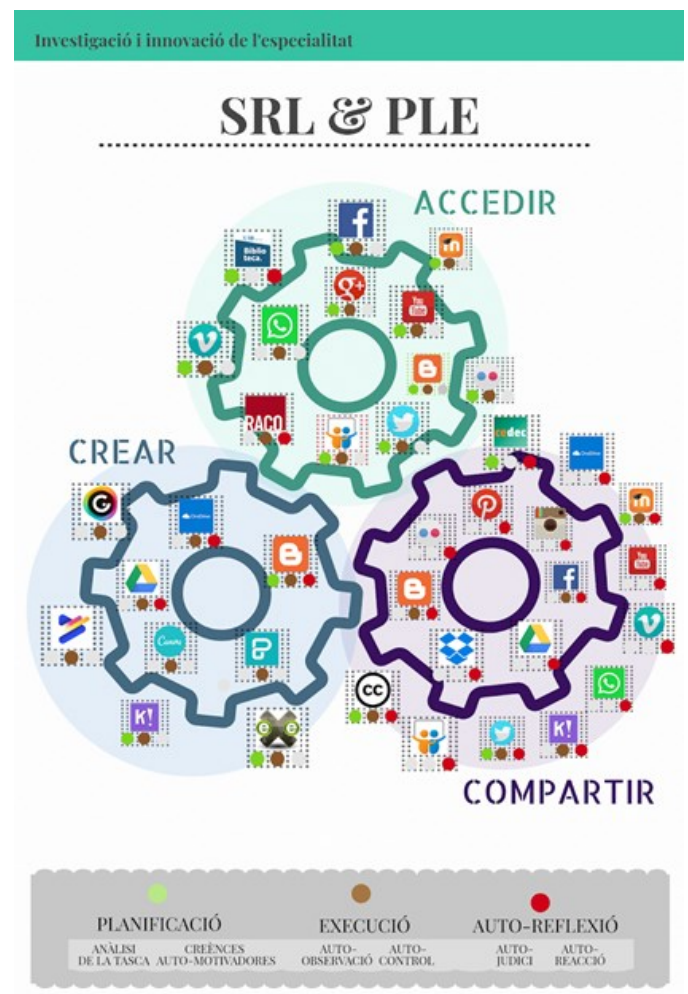

Figura 7. Ejemplo de PLE en diagrama. Grupo 3, Curso 18-19b

\footnotetext{
${ }^{\text {a } U R L: ~}$ https://atreveixteaeducar.blogspot.com/2018/01/ana-riera-torres-16.html

b URL: http://aprenentatgexperiencial.blogspot.com/2019/02/la-utilitat-dun-entorn-personal.html
} 
Al realizar la revisión de los diagramas de PLE (18 en total) que el alumnado creó (como los antes expuestos), se encuentra que Twitter y Blogger están integrados en cada actividad del PLE y en casi cada fase del aprendizaje autorregulado, teniendo en cuenta que Blogger representa la parte reflexiva del eportafolio, mientras que Twitter muestra la parte más social. La siguiente tabla muestra un resumen de los resultados obtenidos (Tabla 5).

Tabla 5. Relación del eportafolio entre el SRL y las actividades del PLE

\begin{tabular}{lllll}
\hline & & \multicolumn{2}{l}{ Fases del aprendizaje autorregulado (SRL) } \\
& & Planificar & Actuar & Reflexionar \\
\hline Actividades del PLE & Acceder & $\begin{array}{l}\text { Twitter } \\
\text { Blogger }\end{array}$ & $\begin{array}{l}\text { Twitter } \\
\text { Blogger }\end{array}$ & $\begin{array}{l}\text { Twitter } \\
\text { Blogger }\end{array}$ \\
& Crear & Blogger & Twitter & Twitter \\
& & Blogger & Blogger \\
& Compartir & Twitter & Twitter & Twitter \\
& & Blogger & Blogger \\
\hline
\end{tabular}

Como se observa en la tabla 4, sólo dos procesos parecen excluyentes. Es decir, con la revisión de todos los diagramas, aparecen las dos plataformas en todos los procesos de PLE y fases del SRL, con dos excepciones. Así, pues, el alumnado sólo llevaría a cabo estrategias de planificación del aprendizaje durante la creación con blogs; mientras que Twitter se emplearía sólo durante las actividades más sociales desarrollando actividades de metacognición relacionadas con la planificación y la motivación inicial.

Algunos fragmentos rescatados de las reflexiones del alumnado permiten conocer algunos matices en relación con el rol de Blogger y Twitter en la intersección entre el PLE y el SRL que se han representado en los diagramas. Por ejemplo, en el siguiente caso, se atribuyen las mismas características a Twitter y Blogger:

[...] la función de las plataformas de Twitter y Blogger van más allá, ya que comparten la característica que, además de crear información, la difundimos al instante con nuestros seguidores o lectores. Este aspecto [...] es el que más aporta a nuestro aprendizaje autorregulado. (Grupo 1, curso 16-17)

En el siguiente caso, se incide en Blogger para planificar mientras se accede y se comparte:

Planificar nuestro trabajo (obteniendo información interesante de nuestros propios compañeros y de autores destacados) y a profundizar ideas o conceptos de la asignatura (desarrollando nuestro aprendizaje y reflexionar sobre él) para después compartirlo con otras personas. (Grupo 2 , curso $17-18)^{d}$

Seguidamente, se selecciona un fragmento sobre Twitter en el que se destaca su valor para la reflexión mientras se accede y se comparte información:

[...] cuando la utilizo para informarme, es decir, para Acceder, me hace reflexionar. Pero cuando pasa a la columna del Crear, es decir, cuando yo lo uso TWITTER para general material, para

\footnotetext{
c URL: http://elracodelprofessor.blogspot.com/2017/02/las-social-media-en-nuestro-aprendizaje.html

d URL: http://ensenyaresferpersones/liures.blogspot.com/
} 
UTE. Revista de Ciències de l'Educació Monogràfic 2020. Pàg. 83-101
El aprendizaje autorregulado en el PLE a través de una estrategia didáctica basada en portafolios con blogs y microblogs

proponer cosas, también lleva enlazada la idea de reflexionar mientras lo hago, de sumar ideas, de modificar posicionamientos, a la hora que genero aquella nuevo producto. $Y$ cuando publico y comparto un tuit propio o leo el de otra persona, aunque impera el momento de reflexión, también invita a la creación, es como un proceso circular, todo aporta posibilidades de nuevas interconexiones. He elegido el TWITTER para desarrollar la idea de multidimensionalidad del PLE, porque es una herramienta que se puede utilizar de muchas formas, y también con objetivos e intenciones diferenciadas. Que genera procesos bidireccionales, donde estamos interconectando fases e intenciones, pero también invita al multi-trayecto, a esta idea de "navegar" de un enlace a otro, de una información a otra, de una idea a otra. (Grupo 4, curso $16-17)^{\mathrm{e}}$

Cabe destacar que, en muchos casos, el alumnado proyecta la experiencia en su futura docencia. Aunque eso son perspectivas que la investigación ha destacado en numerosas ocasiones previamente, el valor añadido de esta actividad permite que se proyecte no sólo la perspectiva PLE en su futura docencias sino además las habilidades metacognitivas del aprendizaje autorregulado.

\section{Discusión y conclusiones}

En este trabajo se ha explorado cómo el profesorado en formación autorregula su entorno personal de aprendizaje (PLE) a través del uso de eportafolios integrados por blogs y microblogs.

La propuesta de diseño didáctico de eportafolio con blogs y microblogs para la creación y reflexión de un yo sinfónico y la colaboración de un yo en red (Cambridge, 2010) parece que se confirma en los diagramas del alumnado. La presencia casi generalizada de las dos plataformas en todos los procesos validarían casi cualquier diseño didáctico, y sin embargo, la presencia exclusiva en dos procesos concretos nos permite confirmar la tendencia hacia nuestra propuesta inicial. Así, pues, que Blogger esté en el proceso de creación y con estrategias metacognitivas para la planificación nos permite confirmar su rol óptimo para una identidad que crea, organiza y cohesiona su aprendizaje; y la presencia de Twitter en el proceso de colaboración también nos hace ver la tendencia de esta plataforma hacia una identidad en red y conectada. Las percepciones del alumnado también van en esa línea como se puede ver a continuación.

Twitter y Blogger suelen tener un valor pedagógico bien sustentado en la literatura y se mencionan sus múltiples beneficios para el autoaprendizaje (Chawinga, 2017), a pesar de que dentro del enfoque PLE se han documentado dificultades para la integración de estrategias de aprendizaje con Twitter (RamírezMera y Tur, 2019). En general, los resultados de esta investigación con enfoque cuantitativo muestran que Blogger es ampliamente aceptado para todas las fases del aprendizaje autorregulado, por lo que es útil en el desarrollo de estrategias metacognitivas de planificación, ejecución y autorreflexión. Sin embargo, el uso de Twitter es una herramienta menos aceptada por el alumnado, con índices menores en todas estas fases, y ligeramente mejor en las dos primeras fases en comparación a la tercera fase de autorreflexión. Es decir, que el alumnado usaría Twitter para estrategias metacognitivas que tengan que ver con la planificación, la automotivación, el control y monitorización del aprendizaje con mayor frecuencia que para la reflexión sobre todo el proceso. Por tanto, aquellos procesos para los que Blogger y Twitter aportan similares funcionalidades son los procesos metacognitivos relacionados con la planificación: establecer objetivos, planificar estrategias, identificar expectativas de autoeficacia y resultados, identificar el valor o el interés en la tarea y orientar metas y propósitos de aprendizaje, y los procesos para implementar y monitorizar el aprendizaje según ese plan y actitud. En consecuencia, aquellos en los que aportarían características diferentes serían en los que tienen que ver con la reflexión

\footnotetext{
e URL: http://metaeducacio.blogspot.com/2017/01/el-nostre-ple.html
} 
metacognitiva sobre el aprendizaje. Por todo ello, la revisión general de los datos cuantitativos confirma una propuesta didáctica que se basa en la construcción del aprendizaje en el yo armónico con Blogger y el yo en red en revisión constante colaborando con otros gracias a Twitter.

Además, esta investigación aporta datos que permiten entender la percepción de ambas plataformas para el aprendizaje autorregulado con cierto detalle. Con base en el test de correlaciones, se encuentra que Twitter y Blogger tienen una alta asociación en las diversas fases del aprendizaje autorregulado, aspecto que permite confirmar que estas dos herramientas contribuyen de manera relevante a la creación de eportafolios y para el autoaprendizaje, como se ha visto en investigaciones empíricas anteriores, tanto en las que las abordan de manera conjunta (Chawinga, 2017) como en las que abordan sus posibilidades en estudios diferenciados, por ejemplo, Biberman-Shalev (2018) sobre los blogs reflexivos, o Tur et al. (2017) sobre las posibilidades de Twitter para la reflexión. Un análisis detallado nos permite ver que Blogger durante la fase de reflexión no tiene una asociación con Twitter en las fases de planificación y ejecución. Además, mientras Twitter tiene sus valores más bajos durante las subfases de auto-control y auto-juicio (que pertenecen a la fase de ejecución y autorreflexión), Blogger mantiene porcentajes altos. Aunque con estos datos no se puede profundizar en este fenómeno, este hecho nos podría indicar el punto clave para el diseño de estrategias en la que se integren ambas herramientas en el PLE, y evitar actividades didácticas en las que ambas herramientas no convergen durante estas fases, incluyendo apoyar el desarrollo de habilidades para el auto-control y auto-juicio durante el uso de Twitter.

Gracias al valor añadido de la actividad de diagrama y los textos reflexivos que el alumnado realiza en último lugar, se pueden observar nuevos matices interesantes. En primer lugar, se confirma que el uso de Blogger tiene una apreciación alta y una elevada frecuencia de uso en los diagramas del PLE, como lo demuestran los resultados de Cho y Cho (2013), y en línea con los resultados del cuestionario. Sin embargo, en segundo lugar, cabe destacar que el uso de Twitter tiene un valor importante en la construcción del eportafolio en todos los procesos de PLE y prácticamente en todas las fases del aprendizaje autorregulado a excepción de la fase de planificación. Esto nos permite plantear la hipótesis que el aprendizaje metacognitivo con Twitter es aún hoy una práctica incipiente. Es decir, sólo una parte del alumnado le da un valor cualitativo diferenciado y es capaz de usarlo para optimizar sus procesos reflexivos, aprovechando la brevedad de los textos que permite el resumen hasta lo esencial o, monitorizando el aprendizaje gracias al encuentro con otros.

En cuanto a la formación del profesorado, por un lado, esta experiencia es una contribución al desarrollo del PLE docente, que incluye tanto la visión más tecnológica como las más reflexiva y metacognitiva al incidir en el proceso de SRL mediante la estrategia didáctica con eportafolios sociales (blogs y microblogs), según el modelo de Cambridge (2010). Por otro lado, a través de esta propuesta, podemos sugerir que esta experiencia del profesorado en formación puede tener impacto en su futura docencia, promoviendo que su propio alumnado pueda generar redes de aprendizaje y habilidades para el aprendizaje autorregulado, claves en la competencias para el aprendizaje a lo largo de la vida (GarcíaMartínez et al., 2020).

El diseño de esta investigación tiene un enfoque mixto pero cabe asumir que se basa sobre todo en el análisis de los datos cuantitativos recogidos, que se complementan en menor medida con un análisis cualitativo. Además, el análisis estadístico, debido a sus características y la forma en cómo son respondidos los instrumentos de investigación, muestra percepciones generales al final del proceso de aprendizaje. Para posteriores investigaciones se recomienda la realización de pruebas de pre-test y postest con la finalidad de de conocer cómo ha impactado la estrategia didáctica en la construcción del aprendizaje metacognitivo con blogs y microblogs. Además, será necesario ampliar el análisis cualitativo sistematizando la colección de datos, con un sistema de categorías y códigos que permita conocer con mayor profundidad de matices y narrativas las reflexiones del alumnado. 
UTE. Revista de Ciències de l'Educació Monogràfic 2020. Pàg. 83-101
El aprendizaje autorregulado en el PLE a través de una estrategia didáctica basada en portafolios con blogs y microblogs

Finalmente, esta investigación requiere de nuevas ediciones puesto que la reducida muestra de alumnado participante no permite generalizar resultados. Además, también requiere de nuevas iteraciones e investigaciones para poder mejorar la estrategia docente y profundizar en las estrategias metacognitivas que presentan mayores retos como las que tienen que ver con la reflexión, tanto las más pausada y cohesionadora como la más fluida y distribuida.

\section{Referencias bibliográficas}

Araka, E., Maina, E., Gitonga, R., y Oboko, R. (2020). Research trends in measurement and intervention tools for self-regulated learning for e-learning environments-systematic review (2008-2018). Research and Practice in Technology Enhanced Learning, 15(6). https://doi.org/10.1186/s41039020-00129-5

Area, M., y Sanabria, A. L. (2014). Changing the rules: from textbooks to PLEs / Cambiando las reglas de juego: de los libros de texto al PLE. Cultura y Educación, 26(4), 802-829. https://doi.org/10.1080/11356405.2014.979068

Barroso, J., Cabero, J., y Vázquez, A. (2012). La formación desde la perspectiva de los entornos personales de aprendizaje (PLE). Apertura, 4(1), 6-12. Recuperado desde http://www.udgvirtual.udg.mx/apertura/index.php/apertura/article/view/209/224

Biberman-Shalev, L. (2018). Personal blogs or communal blogs? Pre-service teachers' perceptionsregarding the contribution of these two platforms to their professional development. Teaching and Teacher Education, 69, 253- 262. https://doi.org/10.1016/j.tate.2017.10.006

Blaschke, L. M. (2019). The Pedagogy-Andragogy-Heutagogy Continuum and Technology-Supported Personal Learning Environments. En I. Jung (Ed.), Open and Distance Education Theory Revisited (pp.75-84). https://doi.org/10.1007/978-981-13-7740-2_9

Cambridge, D. (2010). E-portfolios for lifelong learning and assessment. San Francisco: Jossey-Bass.

Carpenter, J. P., Tur, G., y Marín, V. I. (2016). What do USA and Spanish Pre-service Teachers Think about Educational and Professional use of Twitter? A Comparative Study. Teaching and Teacher Education, 60, 131-143. https://doi.org/10.1016/j.tate.2016.08.011

Castañeda, L., y Adell, J. (2013). La anatomía de los PLEs. En L. Castañeda y J. Adell (Eds.), Entornos Personales de Aprendizaje: Claves para el ecosistema educativo en red (pp.11-27). Alcoy: Marfil.

Castañeda, L., Tur, G. Torres-Kompen, R. (2019). Impacto del concepto PLE en la literatura sobre educación: la última década. RIED, Revista de Iberoamericana de Educación a Distancia, 22(1), pp. 221-241. http://dx.doi.org/10.5944/ ried.22.1.22079

Cebrian, M. (2011). Los ePortafolios en la supervisión del Practicum: modelos pedagógicos y soportes tecnoloógicos. Profesorado, Revista de Curriculum y formación de profesorado, 15(1), 91-107. Recuperado de http://www.ugr.es/ recfpro/rev151ART6.pdf 
Cervantes, V. H. (2005). Interpretación del coeficiente Alpha de Crombach. Avances en Medición, 3, 928. Recuperado desde https://dialnet.unirioja.es/servlet/articulo?codigo $=2300092$

Chaves-Barboza, E., Trujillo-Torres, J. M., Hinojo-Lucena, F. J., y Cáceres-Reche, P. (2019). Personal Learning Environments (PLE) on the bachelor's degree in early education at the University of Granada. In P. Novais et al., (Eds.), ISAm/ 2018 (pp. 381-388). https://doi.org/10.1007/978-3030-01746-0_45

Cedeño, N. (2012). La investigación mixta, estrategia andragógica fundamental para fortalecer la capacidades intelectuales superiores. Res Non Verba, 2(2), 17-36. Recuperado de http://biblio.ecotec.edu.ec/revista/edicion2/revista_completa.pdf\#page $=18$

Chawinga, W. D. (2017). Taking social media to a university classroom: teaching and learning using Twitter and blogs. IJETHE, 14(3). https://doi.org/10.1186/s41239-017-0041-6

Cho, K., y Cho, M. H. (2013). Training of self-regulated learning skills on a social network system. Social Psychology of Education, 16(4), 617-634. https://doi.org/10.1007/s11218-013-9229-3

Cohen, L., Manion, L., y Morrison, K. (2007). Research Methods in Education. London: Routledge.

Creswell, J., y Clarck, P. (2007). El diseño y la realización de la investigación de métodos mixtos. Nueva York: The Guilford press.

Cobos-Sanchiz, D., Lopez-Meneses, E., y Llorent- Vaquero, M. (2016). Proposal for Didactic Innovation with Electronic Portfolios in Blog Format: A Case Study at a University. Form. Univ., 9(6), 27-42. http://dx.doi.org/10.4067/S0718-50062016000600004.

Dabbagh, N., y Castaneda, L. (2020). The PLE as a framework for developing agency in lifelong learning. Education Technology Research and Development, 68(5). https://doi.org/10.1007/s11423-02009831-z

Dabbagh, N., y Kitasantas, A. (2012). Personal Learning Environments, social media, and self-regulated learning: A natural formula for connecting formal and informal learning. Internet and Higher Education, 15, 3-8. https://doi.org/10.1016/j.iheduc.2011.06.002

Dabbagh, N. y Kitsantas, A. (2013). The role of social media in self-regulated learning. International Journal of Web Based Communities, 9(2), 256-273. https://doi.org/10.1504/JJWBC.2013.053248

Dippold, D. (2009). Peer feedback through blogs: Student and teacher perceptions in an Advanced German class. ReCALL- European Association for Computer Assisted Language Learning, 21(1), 18-36. https://doi.org/10.1017/S095834400900010X

Elia, G., y Poce, A. (2010). Future trends for "i-Learning" Experiences. En G. Elia y A. Poce (Eds.), Open Networked "i-Learning" (pp.133-157). Springer: Boston. https://doi.org/10.1007/978-1-44196854-8

Flick, U. (1998). An Introduction to Qualitative Research. London: Sage.

Fiedler, S. H., y Väljataga, T. (2020). Modeling the personal adult learner: the concept of PLE reinterpreted. Interactive Learning Environments, 28(6), 658-670. 
García-Martínez, J.-A., González-Sanmamed, M., y Muñoz-Carril, P.-C. (2020). Entornos personales de aprendizaje: un estudio comparativo entre profesores costarricenses en formación y en ejercicio. Estudios sobre educación, (39), 135-157. https://doi.org/10.15581/004.39.135-157

Korhonen, A.-M., Ruhalahti, S., y Veermans, M. (2018). The online learning process and scaffolding in student teachers' personal learning environments. Education Inf Technol, 24, 755-779. https://doi.org/10.1007/s10639-018-9793-4

Kramarski, B., y Michalsky, T. (2010). Preparing preservice teachers for self-regulated learning in the context of technological pedagogical content knowledge. Learning and Instruction, 20(5), 434447. http://dx.doi.org/10.1016/j.learninstruc.2009.05.003

Krippendorp, K. (2004). Content Analysis: An Introduction to its Methodology. Thousand Oaks, CA: Sage.

Kühn, C. (2017). Are Students Ready to (re)-Design their Personal Learning Environment? The Case of the E-Dynamic.Space. Journal of New Approaches in Education Research, 6(1), 11-19. http://dx.doi.org/10.7821/naer.2017.1.185

Hatzipanagos, S., y Warburton, S. (2009). Feedback as dialogue: exploring the links between formative assessment and social software in distance learning. Learning, Media and Technology, 34(1), $45-$ 59. http://dx.doi.org/10.1080/17439880902759919

Marín, V. I. (2020). Research-based learning in education studies: Design inquiry using group ePortfolios based on blogs. Australasian Journal of Educational Technology, 36(1), 1-20. https://doi.org/10.14742/ajet.4523

Marín, V., Salinas, J., y de Benito, B. (2013). Research results of two personal learning environments experiments in a higher education institution. Interactive learning environments, 22, 205-220. http://dx.doi.org/10.1080/10494820.2013.788031

Marín, V.I. y Tur, G. (2014). Student Teachers' Attitude towards Twitter for Educational Aims. Open Praxis, 6(3), 275-285. http://dx.doi.org/10.5944/openpraxis.6.3.125

Martínez, C., Nocito, G., y Ciesielkiewicz, M. ( 2015). Blogs as a Tool for the Development of SelfRegulated Learning Skills: A Project. American Journal of Educational Research, 3(1), 38-42. Recuperado de http://pubs.sciepub.com/education/3/1/8

Mayring, P. (2004) Qualitative content analysis. En U. Flick, E. von Kardoff y I. Steinke (Eds.), A Companion to Qualitative Research (pp.266-269). London: Sage.

Nkwenti, M. (2016). Design and development of a personal learning environment for corporate Selfregulated learning. Journal of computer and communications, 4, 1-9. http://dx.doi.org/10.4236/jcc.2016.44001

Panagiotidis, P. (2012). Personal Learning Environments for languages learning. Social Technologies, 2(2), 420-440. Recuperado desde http://bit.ly/2JyfeKE

Perera, U., y Gardner, L. (2017). Longitudinal Analysis of Reciprocal Relationships between Digital Literacy and Self-Regulated Learning within Personal Learning Environments. En PACIS 2018 Proceedings. Recuperado desde https://aisel.aisnet.org/pacis2018/157 
Pérez-Garcias, A., Marín, V.I. y Tur, G. (2018). Information Management Tools for the Development of Self-Regulated Learning Skills in Pre-service Teacher Education. @tic revista d'innovació educativa, 21, 10-18. https://doi.org/10.7203/ attic.21.12134

Pozos, K., y Tejeda, J. (2018). Competencias Digitales en Docentes de Educación Superior. Niveles de Dominio y Necesidades Formativas. RIDU, 12(2), 59-87.

http://dx.doi.org/10.19083/ridu.2018.712

Ramírez-Mera, U. y Tur, G. (2019). Seguridad y fiabilidad en la gestión de la información de los entornos personales de aprendizaje (PLE) en la Educación Superior. EDUTEC, 70, 18-33. https://doi.org/10.21556/edutec.2019.70.1435

Romero, L., Saucedo, C., Caliusco, M. L., y Gutiérrez, M. (2019). Supporting self-regulated learning and personalization using ePortfolios: a semantic approach based on learning paths. International Journal of Education Technology Higher Education, 16. https://doi.org/10.1186/s41239-0190146-1

Sabariego, M., Massot, I., y Dorio, I. (2009). Métodos de la investigación cualitativa. En R. Bisquerra (Coord.), Metodología de la investigación educativa (pp. 275-292). Madrid: La Muralla S.A.

Soria, V., y Carrió, M. (2016). Pedagogías disruptivas para la formación inicial de profesorado: usando blogs como e-portafolio. Profesorado, revista de Curriculum y formación del profesorado, 20(2), 382-398. Recuperado de https://recyt.fecyt.es/index.php/profesorado/article/view/52112/31794

Torrado, M. (2009). Estudios de encuentra. En R. Bisquerra (Coord.), Metodología de la investigación educativa (pp. 231-258). Madrid: La Muralla S.A

Torres, R., Edirisingha, P., Canaleta, X., Alsina, M., y Monguet, J. M. (2019). Personal Learning Environments base don Web 2.0 services in higher education. Telematics and informatics, 38, 194-206. https://doi.org/10.1016/j.tele.2018.10.003

Tur, G., Marín, V. I. y Carpenter, J. (2017). Using Twitter in Higher Education in Spain and the USA [Uso de Twitter en educación superior en España y Estados Unidos]. Comunicar, 51. https://doi.org/10.3916/C51-2017-02

Tur, G; Urbina, S. (2016). La colaboración en eportafolios con herramientas de la Web 2.0 en la formación docente inicial. Cultura y Educación: Culture and Education28(3), 601-632. https://doi.org/10.1080/11356405.2016.1203528

Wilson, N., y McLean, S. (1994). Questionnaire Design: A Practical Introduction. Newtown Abbey: University of Ulster Press.

Yen, C.-J., Tu, C.-H., Sujo-Montes, L., y Sealander, K. (2016). A predictor for PLE management: Impacts of self-regulated online learning on students' learning skills. Journal of Educational Technology Development \& Exchange, 9(1), 29-48.

Zimmerman, B. J. (2002). Becoming a self-regulated learner: An overview. Theory Into Practice, 41(2), 64-70. https://doi.org/10.1207/s15430421tip4102_2

Zubizarreta, J. (2009). The learning portfolio. San Francisco: Anker. 
UTE. Revista de Ciències de l'Educació Monogràfic 2020. Pàg. 83-101
El aprendizaje autorregulado en el PLE a través de una estrategia didáctica basada en portafolios con blogs y microblogs

\section{Agradecimientos}

Este estudio ha sido financiado por el Ministerio de Ciencia e Innovación, código de proyecto EDU201784223-R 